\section{Anticoagulants versus antiplatelets for carotid artery dissection}

\author{
Natan M. Bornstein
}

Published online: 5 January 2013

(C) Springer-Verlag Wien 2013

Dissection of the carotid artery is a relatively uncommon etiology of stroke but is a leading cause of ischemic brain events in the young.

Antithrombotic therapy is a common practice and wildly used by neurologists and stroke physicians.

Anticoagulants are the most commonly used antithrombotic treatment for acute carotid artery dissection. However, this policy is not based on randomized controlled studies and in fact a meta-analysis of all the current available data could not demonstrate superiority of anticoagulants over antiplatelets. The published guidelines support this approach and state that antiplatelets and anticoagulants are associated with similar risk of recurrent stroke.

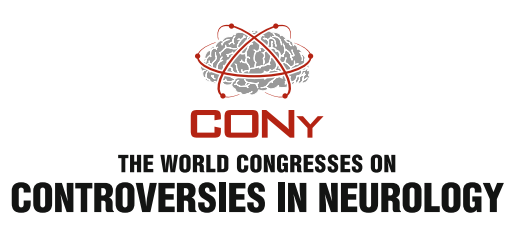

Both authors have agreed that subjective data from observational studies or RCTs do not support the common practice of using anticoagulants over antiplatelets in acute carotid artery dissection, and agreed that large randomized controlled trials are needed to solve this debate.

My personal practice is that in the early immediate period after the initial event anticoagulants are recommended to prevent reoccurrence. However, if the onset of the event occurred more than 24-48 $\mathrm{h}$ prior to the arrival at the emergency room and the neurological condition is stable, antiplatelets are sufficient for secondary stroke prevention.
N. M. Bornstein $(\bowtie)$

Head of Stroke Unit, Department of Neurology,

Sackler Faculty of Medicine, Tel-Aviv Medical Center,

Tel-Aviv University, Tel Aviv, Israel

e-mail: natanb@tlvmc.gov.il 